\title{
Positionspapier zur Diagnostik und Therapie schlafbezogener Atmungsstörungen bei Erwachsenen *
}

\author{
Position Paper on the Diagnosis and Treatment of Breathing Disturbances \\ During Sleep
}

Deutsche Gesellschaft für Pneumologie und Beatmungsmedizin (DGP), Deutsche Gesellschaft für Schlafforschung und Schlafmedizin (DGSM), Verband Pneumologischer Kliniken (VPK) und Bundesverband der Pneumologen (BdP) ${ }^{1}$

Bibliografie

DOI http://dx.doi.org/

$10.1055 / \mathrm{s}-0033-1359183$

Pneumologie 2014; 68: 15-18

(c) Georg Thieme Verlag KG

Stuttgart · New York

ISSN 0934-8387

Korrespondenzanschrift

Prof. Dr. med. Winfried

Randerath

Krankenhaus Bethanien gGmbH

Aufderhöher Str. 169-175

42699 Solingen

randerath@klinik-bethanien.de

\section{Zusammenfassung}

$\nabla$

Schlafbezogene Atmungsstörungen beeinflussen Lebensqualität, Morbidität und Mortalität betroffener Patienten in erheblichem Maße. Daneben haben sie auch wegen ihrer Prävalenz hohe Relevanz für das Gesundheitswesen und die Volkswirtschaft. Die Deutsche Gesellschaft für Pneumologie und Beatmungsmedizin (DGP), die Deutsche Gesellschaft für Schlafforschung und Schlafmedizin (DGSM), der Verband Pneumologischer Kliniken (VPK) und der Bundesverband der Pneumologen (BdP) stellen medizinische Fachgesellschaften und Organisationen dar, deren Mitglieder sich unter anderem schwerpunktmäßig mit der Diagnostik und Therapie schlafbezogener Atmungsstörungen beschäftigen. Aufgrund der aktuellen Entwicklungen in der Versorgung von Patienten mit SBAS sehen sie erhebliche Eingriffe in die Qualität der Betreuung, in der Therapieeinleitung, in der Versorgung mit Hilfsmitteln, in der Nachsorge und in der Wahrnehmung ärztlicher Aufgaben. Die Gesellschaften halten es daher für unumgänglich, dieses gemeinsame Positionspapier zu veröffentlichen. Es basiert auf der intensiven Würdigung und Diskussion der wissenschaftlichen Literatur sowie der klinischen Praxis und Konsensbildung in einer Expertenkommission, die in ausführlicher Darstellung zeitgleich veröffentlicht wird (Randerath et al., Pneumologie 2/2014, Somnologie 1/2014).
* Dieses Positionspapier erscheint zeitgleich in den Fachzeitschriften „Pneumologie“ und „Somnologie“.

\section{Abstract \\ $\nabla$}

Sleep related breathing disorders (SRBD) impair quality of live, morbidity and mortality of affected patients seriously. Moreover, due to their high prevalence, they have a huge impact on health care systems and national economics. The members of the German Respiratory Society, the German Society of Sleep Research and Sleep Medicine, the Association of Pneumological Clinics and the Association of Pneumologists focus strongly on the diagnosis and treatment of SRBD in their daily work. Prevailing developments in the provision of patients with SRBD, have strong impact on the quality of diagnosis and treatment, on the supply of devices, on the follow-up care and on the role and duties of the physician. Therefore, the societies estimate it essential, to publish this common position paper based on the evaluation and discussion of the scientific literature, the clinical practice and a consensus process of an expert group which is published in more detail (Randerath et al., Pneumologie 2/2014, Somnologie 1/2014).

\footnotetext{
${ }^{1}$ Den Sprechern der AG Chirurgische Therapieverfahren in der Schlafmedizin und der Deutschen Gesellschaft Zahnärztliche Schlafmedizin wurde der Text zur Kommentierung vorgelegt.
} 


\section{Relevanz schlafbezogener Atmungsstörungen}

$\nabla$

1. Schlafbezogene Atmungsstörungen haben hohe Bedeutung für den einzelnen Patienten und die Gesellschaft, da sie

a) unabhängiger Risikofaktor u.a. für arterielle Hypertonie, Vorhofflimmern, Herzinsuffizienz, Schlaganfall, Diabetes mellitus, metabolisches Syndrom sind,

b) die Mortalität erhöhen,

c) die Leistungsfähigkeit am Arbeitsplatz und im Straßenverkehr beeinträchtigen,

d) zu erheblichen direkten und indirekten Kosten durch Folgeerkrankungen, Unfälle, Arbeitsausfall und die Behandlung führen.

2. Die medizinisch-wissenschaftliche Entwicklung ist durch die Differenzierung unterschiedlicher Phänotypen von SBAS und die Evaluation technischer Weiterentwicklungen geprägt. Obstruktive und zentrale SBAS und ihre Unterformen müssen daher voneinander und von Hypoventilationssyndromen, auch vom Obesitas-Hypoventilationssyndrom, differenziert werden.

3. Die Diagnostik und Therapie schlafbezogener Atmungsstörungen stellen einen wichtigen Teil der Arbeit niedergelassener und klinisch tätiger Pneumologen dar und sind ein integraler Bestandteil der pneumologischen Weiterbildung. In der interdisziplinären Schlafmedizin arbeiten u.a. Neurologen, Psychiater, Pädiater, Hals-Nasen-Ohren-Ärzte, MundKiefer-Gesichtschirurgen und Zahnärzte zusammen.

\section{Sektorübergreifende Versorgung}

4. Der Grundsatz „ambulant vor stationär“ wird prinzipiell auch für die schlafmedizinische Versorgung zur Anwendung gebracht. Dies hat dazu geführt, dass in den letzten Jahren zunehmend stationär erbrachte Leistungen in Zusammenhang mit der Polysomnografie nicht vergütet wurden. Die Begriffe „ambulant“ und „stationär“ sind für die Betreuung im Schlaflabor jedoch nicht geeignet. Die Art und der Aufwand der Betreuung mit vollständiger nächtlicher apparativer und persönlicher Überwachung, die Anwendung von Geräten, die mit der Atmung interferieren, und die Durchführung in Einrichtungen mit unmittelbarer ärztlicher Erreichbarkeit unterscheiden sich - sofern die Qualitätsvorgaben der Fachgesellschaften eingehalten werden - kaum zwischen der Versorgung im System der kassenärztlichen oder Krankenhausversorgung. Die Bevorzugung eines Systems ist daher nicht gerechtfertigt. Andererseits ist die Möglichkeit der Differenzialtherapie bei Overlap-Syndrom (Koexistenz von COPD und OSAS), die über die Behandlung von schlafbezogenen Atmungsstörungen hinausgeht, durch den Erlaubnisvorbehalt in der ambulanten Versorgung beschränkt. Sofern notwendige persönliche und technische Voraussetzungen gegeben sind, ist dieser Vorbehalt medizinisch nicht nachvollziehbar.

5. Es ist sinnvoll, unter gleichen Bedingungen sektorübergreifende schlafmedizinische Zentren zu ermöglichen, die das gesamte Repertoire diagnostischer Möglichkeiten nach individueller Notwendigkeit einsetzen können.

Dazu ist eine gemeinsame Qualitätssicherung notwendig.

Als Basis für sektorübergreifende Zentren kommen unter anderem Verträge der integrierten Versorgung oder der zwischen den Kostenträgern und der Bayerischen Krankenhaus- gesellschaft abgeschlossene Vertrag in Frage, bei denen die Diagnostik in stationärer, prästationärer und ambulanter Versorgung geregelt ist.

Die Regelungen müssen auch im ambulanten Bereich die Diagnostik und Therapieeinleitung nach den medizinischwissenschaftlichen Voraussetzungen ermöglichen.

6. Der Beschluss des Gemeinsamen Bundesausschusses (GBA) von 2004 sollte zur Verbesserung der Patientenversorgung folgendermaßen angepasst werden:

a) Das bisherige, starr vorgegebene diagnostische und therapeutische Prozedere muss unterschiedlichen Risikosituationen und Krankheitsbildern angepasst werden.

b) Nachuntersuchungen zur Kontrolle der Therapie sollen regelmäßig erfolgen. In Analogie zur regelmäßigen Therapiekontrolle bei anderen technologiebasierten Therapien (wie z.B. bei der Implantation von Herzschrittmachern) und angesichts der Bedeutung der Patientenadhärenz erscheint der Nutzen regelmäßiger Nachkontrollen evident. Die Etablierung einer Versorgungsforschung ist jedoch notwendig.

\section{Diagnostischer Algorithmus nach Vortestwahrscheinlichkeit}

$\nabla$

7. Die diagnostischen Verfahren sollen nach Screening, Bestätigungsdiagnostik und Differenzialdiagnostik unterteilt werden.

a) Unter Screening ist ein Suchtest bei asymptomatischen Patienten mit kardiovaskulären oder metabolischen Risikokonstellationen zu verstehen.

b) Mit der Bestätigungsdiagnostik ist eine Erkrankung bei hoher Vortestwahrscheinlichkeit nachzuweisen.

c) Zur Differenzialdiagnose dienen Polysomnografie (PSG) und erweiterte PSG, mit denen SBAS und andere somnologische Erkrankungen definitiv nachgewiesen oder ausgeschlossen werden.

8. Grundlage für den weiteren Verlauf des Patienten durch Diagnostik und Therapie ist die Einteilung der Krankheitsbilder nach

a) hoher Vortestwahrscheinlichkeit für das Vorliegen obstruktiver SBAS,

b) hoher Vortestwahrscheinlichkeit für einfaches Schnarchen,

c) niedriger Vortestwahrscheinlichkeit für die genannten Störungen und

d) asymptomatischen Patienten mit Risikokonstellation.

Eine hohe Vortestwahrscheinlichkeit für das Vorliegen obstruktiver SBAS liegt vor beim Zutreffen aller der folgenden Kriterien: Schnarchen, (fremdbeobachtete) Atmungsunregelmäßigkeiten im Schlaf und Tagesschläfrigkeit.

Eine hohe Vortestwahrscheinlichkeit für das Vorliegen für einfaches Schnarchen liegt nur vor, wenn der Betroffene zwar schnarcht, aber weder Atmungsunregelmäßigkeiten beobachtet wurden noch Tagessymptome noch kardiovaskuläre oder metabolische Erkrankungen bestehen.

Liegen jeweils nicht alle Kriterien vor, so besteht eine niedrige Vortestwahrscheinlichkeit.

Die Gruppe der asymptomatischen Patienten mit Risikokonstellation beinhaltet Patienten, die unter arterieller Hypertonie, Herzinsuffizienz, Vorhofflimmern, ischämischen zerebrovaskulären Erkrankungen oder Diabetes mellitus leiden, 
aber bei denen weder Tagesschläfrigkeit noch neurokognitive Defizite noch Schnarchen noch Atmungsunregelmäßigkeiten im Schlaf bestehen.

9. Bei hoher Vortestwahrscheinlichkeit für das Vorliegen von OSAS kann die Therapieeinleitung unmittelbar nach einer positiven Polygrafie mit Aufzeichnung der kardiorespiratorischen Parameter mit 6 Meßkanälen erfolgen. Sie wird von einem schlafmedizinisch qualifizierten Arzt nach den Qualitätskriterien der Fachgesellschaften/GBA durchgeführt und manuell und visuell ausgewertet.

Der Verweis auf die Polygrafie darf jedoch nicht zu Zeitverzögerungen in der Diagnostik bei Patienten mit hoher Vortestwahrscheinlichkeit führen, da sie aufgrund ihrer Tagesschläfrigkeit als stark unfallgefährdet und in der Leistungsfähigkeit im täglichen Leben allgemein, am Arbeitsplatz und in der Schule eingeschränkt anzusehen sind. Nicht selten bestehen mehrmonatige Wartezeiten für die Durchführung der Polygrafie. Die unmittelbare Zuweisung zu einem Schlaflabor kann daher bei hoher Vortestwahrscheinlichkeit in begründeten Ausnahmefällen sinnvoll sein, wenn dadurch die Therapieeinleitung beschleunigt werden kann.

Die Einstellung der Therapie erfolgt im Schlaflabor unter polysomnografischer Kontrolle.

10. Die diagnostische Polysomnografie ist notwendig

a) bei niedriger Vortestwahrscheinlichkeit für obstruktive SBAS und einfaches Schnarchen,

b) zur Differenzialdiagnose,

c) bei Diskrepanz zwischen Anamnese und Polygrafie,

d) zur Differenzierung von zentralem und obstruktivem SAS,

e) bei unklarem Schweregrad und Phänotyp der SBAS in der Polygrafie.

11. Arterielle Hypertonie mit fehlender nächtlicher Druckabsenkung oder therapierefraktäre arterielle Hypertonie, Vorhofflimmern, Herzinsuffizienz, koronare Herzkrankheit, zerebrovaskuläre Erkrankungen und Diabetes mellitus gehören zu Erkrankungen, die häufig mit schlafbezogenen Atmungsstörungen assoziiert sind. Wegen der hohen Prävalenz und des Risikos einer Prognoseverschlechterung ist bei Vorliegen dieser Erkrankungen nach schlafbezogenen Atmungsstörungen gezielt zu suchen.

Bestehen zusätzliche Symptome, die bereits auf schlafbezogene Atmungsstörungen hinweisen, ist entsprechend der Vortestwahrscheinlichkeit eine Polygrafie (6 Kanäle) oder Polysomnografie nach den oben beschriebenen Grundsätzen durchzuführen.

Bestehen weder Tagesschläfrigkeit noch neurokognitive Defizite noch Schnarchen noch Atmungsunregelmäßigkeiten im Schlaf (asymptomatische Patienten mit Risikokonstellation), so ist als Suchtest ein 1- oder 2-Kanalscreening möglich. Dieser Suchtest kann weder als Bestätigungstest bei schon bestehendem klinischem Verdacht noch zum sicheren Ausschluss oder zur definitiven Differenzialdiagnose eingesetzt werden. Er kann jedoch eine große Zahl bisher unbekannter SBAS in den genannten Risikogruppen finden lassen und so der weiterführenden Diagnostik zuleiten.

Die Anwender dieser Testverfahren müssen eine noch zu implementierende Schulung, Prüfung und Zulassung durchlaufen, ähnlich der NUB-Kurse der Ärztekammern. Des Weiteren muss die Vergütung der Leistung sichergestellt werden.

Wenn sich in einem solchen Screening-Test der V.a. ein Schlafapnoesyndrom erhärtet, muss der Patient einem schlafmedizinisch qualifizierten Arzt zur weiteren Diagnostik vorgestellt werden. Eine direkte Einweisung in die höchste Versorgungsebene ist nicht zielführend.

\section{Therapie, Versorgung und Nachkontrolle}

$\nabla$

12. Die Positivdrucktherapie stellt den Therapiestandard bei Patienten mit OSAS dar, da sie den besten Effekt auf Symptomatik und Prognose zeigt.

13. Zur Ersteinstellung gehört eine strukturierte Patientenschulung durch das versorgende schlafmedizinische Zentrum.

14. Aus Compliance-Gründen ist eine unmittelbare Versorgung mit dem definitiven Therapiegerät noch im Schlaflabor notwendig.

Die Auswahl des Gerätes und der Maske sowie die Erstanpassung derselben gehören in die Hand des Schlafmediziners und seiner geschulten Mitarbeiter.

Eine Zeitverzögerung durch Genehmigungsprozesse oder die Geräteversorgung ist auszuschließen, da gerade die ersten Tage über die Langzeit-Nutzung entscheiden.

Die gesetzlichen Vorgaben des $§ 128$ SGB V sollten entsprechend angepasst werden.

15. Eine erste Kontrolle soll nach 2-6 Wochen unter Zuhilfenahme zumindest einer 6-Kanal-Polygrafie, weitere Kontrollen im Abstand von je einem Jahr erfolgen. Die Kontrollen erfolgen in standardisierter Weise und beinhalten die Erfassung der Atmung im Schlaf, der Nutzung, der Tagessymptomatik, der Gerätefunktion, der Maske und des Interface. Vorzeitige Kontrollen sind bei Veränderungen der Indikation oder ungenügender Wirksamkeit notwendig.

Auf unterjährige Kontrollen kann bei unkompliziertem Verlauf und unveränderter Gesamtsituation im Einzelfall verzichtet werden.

16. Bei einem OSAS mit einem $\mathrm{AHI}<30 / \mathrm{h}$ und einem Body Mass Index $<30 \mathrm{~kg} / \mathrm{m}^{2}$ können Unterkieferprotrusionsschienen (UPS, gemäß Definition AADSM 2013) eingesetzt werden.

a) Die Indikation muss auf einer vollständigen schlafmedizinischen Untersuchung einschließlich PSG basieren, eine alleinige PG reicht nicht aus.

b) Der Patient ist über die Vor- und Nachteile der Positivdrucktherapie und der UPS aufzuklären.

c) Eine Therapie mit UPS aufgrund unzufriedenstellender Positivdruck-Compliance kann bei einem AHI>30/h erst nach Optimierung von Maske und Interface, intensiver erneuter Schulung von Patient und Angehörigen sowie ggf. nach psychiatrischer Diagnostik und Behandlung von Angststörungen eingeleitet werden.

d) Die Indikation muss vom schlafmedizinisch qualifizierten Arzt gestellt werden. Die Versorgung mit einer UPS sollte durch schlafmedizinisch qualifizierte Zahnmediziner erfolgen. Die vorherige Anpassung einer individuell qualifiziert hergestellten Testschiene kann sinnvoll sein. In jedem Fall ist die Überprüfung durch einen schlafmedizinisch geschulten Arzt einschließlich einer nächtlichen Messung (mindestens 6 Kanäle) unverzichtbar.

e) Die Wirksamkeit der Therapie mit UPS muss innerhalb von 1-3 Monate durch einen schlafmedizinisch geschulten Arzt einschließlich einer nächtlichen Messung (mindestens 6 Kanäle) überprüft werden.

f) Die Kostenübernahme durch die Kostenträger einer nach diesen Kriterien angepassten UPS ist zu fordern. 
17. Die Entfernung vergrößerter Gaumenmandeln kann auch beim Erwachsenen in der Therapie des obstruktiven Schlafapnoe-Syndroms im Einzelfall sinnvoll sein.

Darüber hinaus hat sich die maxillo-mandibuläre Osteotomie als chirurgisches Verfahren auch langfristig als effizient erwiesen.

Als neues Verfahren kann im Einzelfall bei einem AHI 15-50/h und einer Adipositas Schweregrad $\leq$ I die unilaterale Stimulation des N. hypoglossus durch implantierbare Generatoren erwogen werden, wenn Positivdruckverfahren und UPS nicht angewandt werden können.

In ausgewählten Fällen können bei einem leichten bis mittelschweren OSAS andere Operationen im Bereich der oberen Atemwege (z.B. Weichgaumen) bei hauptsächlich für das OSAS verantwortlicher Pathologie erwogen werden, wenn konservative Maßnahmen keinen Erfolg gebracht haben.

\section{Ärztliche Aufgaben und Delegation}

18. Zu den ärztlichen Aufgaben des Schlafmediziners gehören

a) Indikationsstellung und Durchführung der Diagnostik und Therapie individuell nach Notwendigkeit des Patienten.

b) Auswahl und Anpassung der geeigneten Therapieform, einschließlich Schulung, Maskenauswahl, -erstanpassung, Geräteauswahl und Geräteeinstellung.

c) Nachbetreuung des Patienten, einschließlich regelmäßiger Kontrollen und Beseitigung von Therapieproblemen und -hindernissen.

19. Einige schlafmedizinische Leistungen können vom Arzt zusammen mit besonders qualifiziertem Personal erbracht werden. Geschultes Personal von Hilfsmittellieferanten kann in die Adaptation von Masken im Langzeitverlauf (nicht in der Erstanpassung) eingebunden sein. Es muss sichergestellt sein, dass Hausarzt und Schlafmediziner über die Intervention informiert werden. Eine Umversorgung von Nasenmaske auf Nasen-Mundmaske oder vice versa bedarf der Kontrolle durch einen schlafmedizinisch qualifizierten Arzt, der über den Umfang notwendiger Untersuchungen entscheidet.

20. Die Rolle des Hausarztes beinhaltet die Erhebung des Verdachtes auf SBAS, die Wertung der Vortestwahrscheinlichkeit und daraus resultierend Lenkung des Patienten, das Screening bei asymptomatischen Patienten mit Risikofaktoren, Wahrnehmung von Therapieproblemen.

\section{Austausch und Umversorgung von Therapiegeräten}

$\nabla$

21. Eine Versorgung darf nur mit Geräten erfolgen, die Minimalkriterien an Druckstabilität, Regelungsverhalten, Trigger, Lautstärke, Durchflussrate und andere Parameter erfüllen (Konsensuspapier).

22. Eine Umversorgung bei APAP-, BPAP-, ASV-Geräten unterschiedlicher Bauart, Typen oder Hersteller ist nur nach Neueinleitung im Schlaflabor möglich. Bei einer Umversorgung von CPAP-Geräten, die die Minimalkriterien erfüllen, ist der betreuende, schlafmedizinisch qualifizierte Arzt zu informieren, der über die Vorgehensweise zu entscheiden hat.

\section{Verträge und Ausschreibungen}

$\nabla$

23. Die schlafmedizinische Versorgung ist in Deutschland inhomogen. Aufgrund der Prävalenz der Erkrankung und der mehrmonatigen Wartezeiten auf eine PSG (bis zu mehr als 12 Monate) ist eine Unterversorgung anzunehmen. Dazu trägt die extrem unterschiedliche Vergütungsstruktur für schlafmedizinische Leistungen bei. Das Umfeld ist auch von neuen Vertragsformen und Hilfsmittelausschreibungen geprägt.

24. Der Versuch der Minimierung der Diagnostik und Therapieeinleitung wird der Krankheitssituation zahlreicher schlafgestörter Patienten nicht gerecht. Die Antizipation der Diagnose schlafbezogener Atmungsstörungen erschwert häufig die Erkennung anderer Ursachen des Leitsymptoms des nicht-erholsamen Schlafes.

25. Die Betreuung des Schlafapnoe-Patienten ist jedoch nicht standardisierbar, beinhaltet einen hohen Anteil an personenbezogenen Dienstleistungen und ist übergreifend. Eine ineffektive Therapie oder der Behandlungsabbruch stellt ein gesundheitliches Risiko sowohl kurz- als auch langfristig dar. Damit sind Ausschreibungen für die Versorgung bei schlafbezogenen Atmungsstörungen ungeeignet. Auch bei der Gestaltung anderer Vertragsformen sind die in diesem Papier dargelegten Grundsätze zu berücksichtigen.

Eine flächendeckende und gleichzeitig wirtschaftliche Versorgung der Bevölkerung bedarf neuer Regeln. Die bisherigen Konzepte haben zu einer inhomogenen Versorgungslage geführt und werden dem Patienteninteresse nach einer qualitativ hochwertigen und wohnortnahen Versorgung nicht gerecht.

Eine ausreichende Versorgung mit schlafmedizinischen Leistungen ist deshalb auch nach der Ausweitung der PSG im ambulanten Bereich noch nicht abzusehen. Um ein flächendeckendes Versorgungsangebot machen zu können, ist eine sektorenübergreifende Bedarfssteuerung sinnvoll. Regelungen, die z.B. in der Dialyseversorgung bestehen, könnten als Beispiel dienen.

Voraussetzung jeglicher sektorenübergreifender Versorgung ist eine klare Definition des Versorgungsumfanges, wie im Positionspapier erfolgt, eine gemeinsame Qualitätssicherung und eine gleiche Vergütung gleicher Leistungen. So können Fehlentwicklungen, die ohne Zweifel zu Lasten der Patienten gehen, wie Fallsplitting mit langen Wartezeiten und Risikoselektion sowie Missachtung der differenzialdiagnostischen und differenzialtherapeutischen Breite der Schlafmedizin vermieden werden. 